\title{
Brecha de género en el conocimiento político infantil: influencia de las redes sociales y la socialización política*
}

\author{
Miriam Hernández ${ }^{1}$ \\ Alma Rosa Saldierna-Salas ${ }^{2}$ \\ Carlos Muñiz ${ }^{3}$
}

Felipe de Jesús Marañón-Lazcano ${ }^{4}$

Recibido: 16/02/2019

Aprobado por pares: 14/07/2019
Enviado a pares: $17 / 02 / 2019$

Aceptado: 16/07/2019

DOI: 10.5294/pacla.2020.23.4.3

\section{Para citar este artículo / to reference this article / para citar este artigo}

Hernández, M., Saldierna-Salas, A. R., Muñiz, C. y Marañón-Lazcano, F. de J. (2020). Brecha de género en el conocimiento político infantil: influencia de las redes sociales y la socialización política. Palabra Clave, 23(4), e2343. https://doi.org/10.5294/pacla.2020.23.4.3

\section{Resumen}

El conocimiento político es un determinante esencial de la participación política ciudadana. No obstante, múltiples estudios han señalado que existe una brecha en el conocimiento político de hombres y mujeres. Este trabajo tuvo como objetivo ampliar la investigación de la brecha de género a los niños mexicanos como consecuencia de la socialización política (escolar y familiar) e incluyó como factor de interés el consumo de redes sociales.

\footnotetext{
Artículo realizado con el apoyo financiero de Fondos SEP-CONACYT (clave: 264415). Hace parte del proyecto "La formación cívica de la educación básica como agente de socialización política de los estudiantes: diagnóstico de su impacto en comparación con el desarrollado por la familia y los medios de comunicación”, realizado por la Universidad Autónoma de Nuevo León, Nuevo León, México.

1 https://orcid.org/0000-0003-0872-7514. California State University, Dominguez Hills, Estados Unidos. mihernandez@csudh.edu

2 https://orcid.org/0000-0003-1805-9740. Universidad Autónoma de Nuevo León, México. alma.saldiernasls@uanl.edu.mx

3 https://orcid.org/0000-0002-9021-8198. Universidad Autónoma de Nuevo León, México. carlos.munizm@uanl.mx

4 https://orcid.org/0000-0002-0705-6336. Universidad Autónoma de Nuevo León, México. felipe.maranonlzcn@uanl.edu.mx
} 
Se realizó una encuesta $(n=1539)$ entre abril y mayo 2017 a alumnos de quinto año (10-11 años) y sexto año (12-13 años) de primaria en el estado de Nuevo León (México). Los resultados indicaron que la brecha de género no es materialmente significativa a esta edad, pero ya hay indicios de lo que se observa en la adultez. Los análisis multivariados indicaron que la socialización política y el consumo de redes sociales contribuye de diferente manera al conocimiento político de ambos géneros. El entorno con conversación política mejora el conocimiento político de las niñas, mientras el consumo de información política vía redes sociales lo disminuye. Para ambos grupos, la socialización escolar no tiene ningún efecto a esta edad. Los resultados de este estudio contribuyen a la investigación sobre el conocimiento político en el contexto latinoamericano y reconoce la repercusión política en los años formativos de los ciudadanos. Se discuten implicaciones prácticas de la socialización política y de género, así como el uso de redes sociales en el desarrollo ciudadano de niños y niñas.

\section{Palabras clave (Fuente: tesauro de la Unesco)}

Brecha de género; conocimiento político; redes sociales; medios sociales; socialización familiar; socialización escolar. 


\section{Gender Gap in Children's Political Knowledge: Influence of Social Media and Political Socialization*}

\section{Abstract}

Political knowledge is an essential determining factor of citizen political participation. However, multiple studies have pointed to a gap in the political knowledge of men and women. This paper aims to extend research into the gender gap due to political socialization (school and family) to Mexican children, including social media consumption as a factor of interest. A survey $(\mathrm{n}=1539)$ was conducted between April and May 2017 among fifth (10-11 years) and sixth (12-13 years) graders in Nuevo León (Mexico). The results suggested that the gender gap is not materially significant at this age, but there are already indications of what is observed in adulthood. Multivariate analyses indicated that political socialization and social media consumption contribute differently to both genders' political knowledge. A political conversation environment improves girls' political knowledge, while political information consumption via social media reduces it. School socialization does not affect either group. This study adds to the research on political knowledge in the Latin American context and recognizes the political repercussion in citizens' formative years. The practical implications of political and gender socialization and social media for boys' and girls' citizen development are discussed.

\section{Keywords (Source: Unesco Thesaurus)}

Gender gap; political knowledge; social media; social networks (online); family socialization; school socialization.

Article prepared with the financial support of Fondos SEP-CONACYT (Code: 264415). It is part of the project "La formación cívica de la educación básica como agente de socialización política de los estudiantes: diagnóstico de su impacto en comparación con el desarrollado por la familia y los medios de comunicación," carried out by the Universidad Autónoma de Nuevo León, Nuevo León, Mexico. 


\section{Brecha de gênero no conhecimento político infantil: influência das redes sociais e da socialização política*}

\section{Resumo}

O conhecimento político é um determinante essencial da participação política cidadã. Não obstante, múltiplos estudos têm indicado que existe uma brecha no conhecimento político de homens e mulheres. Este trabalho teve como objetivo ampliar a pesquisa sobre a brecha de gênero nas crianças mexicanas como consequência da socialização política (escolar e familiar) e incluiu como fator de interesse o consumo de redes sociais. Realizou-se um questionário $(\mathrm{n}=1539)$ entre abril e maio de 2017 a alunos de quinto ano (10-11 anos) e sexto ano (12-13 anos) de primária no estado de Nuevo León (México). Os resultados indicaram que a brecha de gênero não é materialmente significativa nessa idade, mas já há indícios do que se observa na idade adulta. As análises multivariadas indicaram que a socialização política e o consumo de redes sociais contribuem de diferente maneira para o conhecimento político de ambos os gêneros. $\mathrm{O}$ ambiente com conversas políticas melhora o conhecimento político das meninas, enquanto o consumo de informação política via redes sociais diminui-o. Para ambos os grupos, a socialização escolar não tem nenhum efeito nessa idade. Os resultados deste estudo contribuem para a pesquisa sobre o conhecimento político no contexto latino-americano e reconhece a repercussão política nos anos formativos dos cidadãos. São discutidas implicações práticas da socialização política e de gênero, bem como o uso de redes sociais no desenvolvimento cidadão de meninos e meninas.

\section{Palavras-chave (Fonte: tesauro da Unesco)}

Diferença de gênero; conhecimento político; redes sociais; mídia social; socialização familiar; socialização escolar

\footnotetext{
* Artigo realizado com o apoio financeiro de Fondos SEP-CONACYT (clave: 264415). Faz parte do projeto "A formação cívica da educação básica como agente de socialização política dos estudantes: diagnóstico de seu impacto em comparação com o desenvolvido pela família e pelos meios de comunicação", realizado pela Universidade Autônoma de Nuevo León, Nuevo León, México.
} 


\section{Introducción}

La brecha en el conocimiento político ${ }^{1}$ entre hombres y mujeres es una constante mundial (Dolan, 2011; Dow, 2009; Fraile, 2014; Hernández y Marañón, 2017; Jerit \& Barabas, 2017; Lizotte \& Sidman, 2009; Mondak \& Anderson, 2004). Estudio tras estudio ha demostrado que las mujeres no solo "saben menos" de hechos políticos, sino que dudan en mayor grado de su capacidad política (Lizotte \& Sidman, 2009; Mondak \& Anderson, 2004) y tienen una tendencia a evadir las discusiones políticas que conducen a un mayor nivel de información pública (Hernández y Marañón, 2017), aun cuando se controlan factores socioeconómicos y demográficos. Esta brecha tiene consecuencias potenciales para la manera en que los ciudadanos resuelven sus dificultades personales y en la que ejercen sus derechos y obligaciones democráticos (Delli \& Keeter, 1996).

Dada la importancia de la sofisticación política y la clara brecha entre hombres y mujeres, la literatura ha investigado qué determina esta diferencia. Por ejemplo, un conocimiento saludable de hechos políticos es resultado de un interés por aprender, la habilidad personal y externa, y oportunidades informativas para hacerlo (Delli \& Keeter, 1996). En las mujeres, los estudios han señalado que las diferencias socioeconómicas (Batista, 2012; Fraile \& Gómez, 2017; Lizotte \& Sidman, 2009; Mondak \& Anderson, 2004) y la socialización de género que reciben (Dow, 2009; Raffaelli \& Ontai, 2004; Sapiro, 2004; Trevor, 1999; Wolak \& McDevitt, 2011) limitan el interés, la habilidad y las oportunidades que tienen para aprender. Sin descontar su contribución, esta investigación se ha centrado principalmente en contextos estadounidenses y europeos, con muy pocos casos en contextos hispanos (para una excepción, véanse Ferrín et al., 2019; Fraile, 2014) o latinoamericanos (Batista, 2012; Fraile \& Gómez, 2017; García, 2015; Hernández y Marañón, 2017). De igual manera, el análisis de la brecha de género se ha limitado a adultos, aunque en los últimos años el interés en el conocimiento político de niños y adolescentes se ha incrementado (Abendschön \& Tausendpfund, 2017; Fraile \& Sánchez-Vítores, 2020; Simon, 2017). Este ejercicio tiene como objetivo extender la investigación de la brecha de género a los niños mexicanos como consecuencia de la socialización política y de género. A la par, en vista de que existe un uso creciente de redes sociales en 
los jóvenes y el acceso a la información ha evolucionado desde los primeros contextos examinados (disponibilidad de información a través de medios digitales), se incluye como factor especial de interés el consumo de redes sociales. Nuestro proyecto se centra en el estado de Nuevo León, uno de los estados de la República Mexicana, donde las diferencias socioeconómicas, educativas y acceso mediático son más constantes, en comparación con otros estados del país.

La primera sección explora brevemente la literatura alrededor de la brecha en el conocimiento político entre hombres y mujeres, mientras que la segunda sección analiza cómo la socialización política y las redes sociales contribuyen a la brecha, así como a la situación existente en México. Estos aspectos servirán para comparar el conocimiento, la socialización política y el uso de redes sociales entre niños y niñas. Los resultados servirán para analizar los factores detrás de la brecha de género y discutir en qué momento se vuelve determinante, así como la manera en que puede ser abordada social y políticamente.

\section{Información política y brecha de género}

El conocimiento político, el rango de información sobre hechos y eventos políticos, es un recurso indispensable para la eficaz ejecución de las responsabilidades de un ciudadano, su empoderamiento y el desarrollo socioeconómico de una sociedad (Delli \& Keeter, 1996). Las personas con un grado más alto de sofisticación política tienen mayor capacidad para alinear conscientemente sus opiniones con la elección de sus representantes y sus opiniones son menos fluctuantes a lo largo del tiempo, o son víctimas de información oportunista (Dassonneville \& McAllister, 2018; Ferrín et al., 2019; Luskin, 1990; Wolak \& McDevitt, 2011). Igualmente, la carencia de información política relevante está relacionada con la inhabilidad para influir en el proceso político y traducir el apoyo político a un candidato en beneficios concretos para el individuo y su comunidad (Fraile \& Gómez, 2015; García, 2015), tal como presentar denuncias, conocer los derechos de los cuales goza, requerir ayuda de la institución adecuada, entre otros.

La adquisición de conocimiento político está directamente ligada a la habilidad personal (confianza del ciudadano en su capacidad indivi- 
dual) y externa (confianza en su capacidad para influir en las instituciones), la motivación política (el interés del individuo en hechos políticos) y la disponibilidad de información (acceso a medios de comunicación libres y competentes, y a información de calidad) (Delli \& Keeter, 1996; Jerit et al., 2006; Luskin, 1990). Estos elementos del modelo motivaciónhabilidad-oportunidad, estudiados en primera instancia por Delli \& Keeter (1996), son afectados por factores estructurales como la clase social (las personas con mayor disponibilidad de recursos pueden aprender más), la edad (a medida que la gente envejece aumenta su conocimiento), la educación (un mayor número de años académicos va ligado a mayor conocimiento) y el género (las mujeres tienen un menor interés y conocimiento político que los hombres).

Dada entonces la importancia de la sofisticación política, la existencia significativa de una brecha de género informativa habla de la desigualdad de circunstancias para las mujeres en adquirirla. Estas diferencias en sofisticación política han sido documentadas continuamente en contextos estadounidenses (Dolan, 2011; Dow, 2009; Jerit \& Barabas, 2017; Lizotte \& Sidman, 2009; Mondak \& Anderson, 2004) y europeos (Fraile, 2014) a lo largo de los años. No obstante, en América Latina, los estudios han sido más reducidos, aunque concurren en que las mujeres tienen un nivel de conocimiento político significativamente más bajo que los hombres. Por ejemplo, Batista (2012) analiza el impacto de esta brecha en la baja participación política de las mujeres en América Latina, mientras que Fraile \& Gómez (2015) comparan 18 países latinoamericanos y confirman una brecha representativa en cada uno de ellos, que se reduce en ámbitos urbanos y de mayor educación. En el contexto mexicano, García (2015) y Hernández y Marañón (2017) corroboran resultados similares. Por ejemplo, en la Encuesta Nacional sobre Cultura Política y Prácticas Ciudadanas (Encup) 2012, el número de respuestas correctas dadas por los hombres es significativamente mayor $\left(M_{\text {mujeres }}=1,86\right.$ preguntas correctas vs. $\left.M_{\text {hombres }}=2,21\right)$ (Hernández y Marañón, 2017). Por su parte, García (2015) traza la brecha de género en las últimas dos décadas y observa que esta es constante, pero se reduce en temas de figuras políticas y años electorales, cuando la información política es más accesible. 
Aparte de los factores estructurales que afectan la capacidad para adquirir sofisticación política, las causas de esta brecha se han atribuido al tipo de socialización política que reciben las mujeres, que impacta su motivación para aprender y su interés en la política (p. ej., "la política es cosa de hombres") (Delli \& Keeter, 1996; Dolan, 2011; Dow, 2009), las hace más aversivas al riesgo (p. ej., responden "no sé" o no responden si no están completamente seguras de tener una respuesta correcta) (Lizotte \& Sidman, 2009; Mondak \& Creel, 2001; Mondak \& Anderson, 2004) y socialmente las confina al ámbito familiar y las desalienta de participar en el ámbito público (Ferrín et al., 2019; Fox \& Lawless, 2004). Otro aspecto que se ha sido estimado influyente en las últimas dos décadas es la adquisición de información por las redes sociales (Bode, 2016; Cacciatore et al., 2018; Gil de Zúñiga et al., 2017; Prior, 2005). Las redes sociales permiten democratizar el acceso a la información y tienen el potencial para disminuir la brecha de género. Las siguientes secciones exploran estos dos últimos componentes que se integran al modelo motivación-habilidad-oportunidad.

\section{Socialización política: escuela, familia y redes sociales}

La socialización política se entiende como la manera en que las sociedades y sus sistemas políticos inculcan normas y prácticas para sus ciudadanos y miembros (Sapiro, 2004). Individualmente, la socialización política estudia los patrones y procesos a través de los cuales las personas aprenden, se comprometen y se desarrollan políticamente. Naturalmente, los estudios referentes a la socialización política se han enfocado en los jóvenes, dado que la adquisición de conocimiento y hábitos políticos en los años formativos es útil para entender la base de las actitudes políticas (valores políticos e identidad partidista), el compromiso político (interés y eficacia política) y los comportamientos políticos (participación política convencional y no convencional) que se desarrollarán en la vida adulta (Ferrín et al., 2019; Fraile \& Sánchez-Vítores, 2019; Nateras, 2003; Neundorf \& Smets, 2020).

Este proceso formativo es una consecuencia directa e indirecta de interacciones con familiares, profesores, contexto político y medios de comunicación. Estas relaciones son el vehículo esencial a través del cual se 
influye, se alienta o se desanima cierto comportamiento político, particularmente entre los 7 y 14 años (Barbeito, 2002; Ferrín et al., 2019). Entre los agentes socializantes más estudiados están los de la escuela y la familia.

La escuela, como se ha indicado, está altamente relacionada con el interés político y, por ende, con el conocimiento político. La educación se ha visto como un elemento similar a la situación socioeconómica: a mayor ingreso familiar, mayor número de años estudiados, interés político y sofisticación política (Delli \& Keeter, 1996; Luskin, 1990). Dentro de la educación, se ha prestado especial atención a la formación cívica —las clases que instruyen sobre el sistema de gobierno y las responsabilidades cívicas de los estudiantes-y su capacidad para promocionar el compromiso político (Neundorf \& Smets, 2017; Torney-Purta et al., 2007). Estos artículos han visto resultados prometedores, especialmente cuando la formación involucra el libre intercambio de opiniones y complementa la socialización política familiar.

Con respecto a la familia, la influencia de los padres en las orientaciones políticas es uno de los determinantes más importantes en el desarrollo de la identificación partidista y el interés político (Barbeito, 2002; Jaime, 2000; Lyons, 2017; Neundorf \& Smets, 2020). La cantidad de tiempo que los niños pasan con sus padres, la cercanía de la relación y la búsqueda de aprobación parental predispone a los niños a ser más receptivos a las características políticas de su contexto familiar (Lyons, 2017). Por ejemplo, en familias altamente politizadas, los padres estimulan el compromiso con eventos cívicos y políticos, con lo que proveen señales para la futura imitación o adopción de actitudes y comportamientos políticos (Neundorf \& Smetz, 2017).

Además, otro fundamento a considerar es la disponibilidad de información. Para tener un índice más alto de conocimiento político, es necesario tener mayor acceso a información política (Delli \& Keeter, 1996; Prior, 2005). Una mayor oferta y facilidad de acceso a datos políticos (en calidad y en cantidad), a través de medios de comunicación tradicionales o redes sociales, mejora el entendimiento político. Sin embargo, a diferencia de ge- 
neraciones pasadas, en las que los medios tradicionales dedicaban una parte esencial de su programación a noticieros y las personas estaban expuestas a información política voluntaria o incidentalmente, y así fomentaban la sofisticación política, los medios actuales dedican un mayor espacio a entretenimiento o infoentretenimiento (Kim et al., 2013; Gil de Zúniga et al., 2017; Lee \& Xenos, 2019; Prior, 2005). Además, el diseño de las redes sociales permite discriminar el tipo de información que se consume, lo cual ha incrementado el acceso al entretenimiento y, por consecuencia, a la información política no fáctica (Cacciatore et al., 2018; Gil de Zúñiga et al., 2017; Lee \& Xenos, 2019; Prior, 2005). Incluso, cuando los estudios han indicado que sí se consume información política a través de plataformas en línea (Bode, 2016; Gil de Zúñiga et al., 2017), estas tienden a aumentar la información partidista o desinformación sobre temas específicos en personas con poco interés político (Bode \& Vraga, 2015). Dado que 99 de cada 100 mexicanos tienen una cuenta de Facebook o algún tipo de red social (Hurtado, 2019), y el consumo de noticias vía redes sociales está en aumento, particularmente entre los jóvenes (De Assis, 2019), su análisis como mediador del conocimiento político resulta indispensable. La inclusión de las redes sociales permite, además, dar vitalidad al modelo tradicional de Delli \& Keeter (1996), en consideración a que la disponibilidad de información tiende a analizarse como una constante en la investigación de los factores que afectan la sofisticación política.

\section{Socialización de género}

Sin descontar el efecto ecualizador que tiene la escuela, los medios de comunicación y la familia como agentes socializantes, no deben descartarse las diferentes expectativas y la división de roles que existen para hombres y mujeres, y que ponen en marcha los distintos niveles de aprendizaje que se ven en la adultez (Ferrín et al., 2014; Raffaelli \& Ontai, 2004; Wolak \& McDevitt, 2010). La socialización de género empieza tempranamente cuando los padres promueven cierto tipo de comportamiento estereotípico (p. ej., figuras de acción o equipo de deporte como juegos para niños y muñecas, miniaturas de cocina y casas para niñas) (Fraile \& Sánchez-Vítores, 2019; Leaper, 2014), y continúa en los años formativos cuando estos jóvenes reciben retroalimentación de sus maestros ("las matemáticas son más fáciles 
para los chicos”, se espera que las chicas sean más serviciales, organizadas o bien portadas) (Bigler et al., 2013), cuando se fomenta la participación pública para los hombres ("la política es cosa de hombres") y cuando se exaltan los valores de esposa/madre para las mujeres (un papel eminentemente privado y pasivo) (Fraile \& Sánchez-Vítores, 2019; García \& Frutos, 1999; Hernández, 2018).

Estos factores tienen implicaciones potenciales para la formación del interés político y, por ende, de la sofisticación política, en la que las mujeres históricamente han tenido niveles más bajos (Fraile \& Sánchez-Vítores, 2019). Por ejemplo, las personas con mayor educación se sienten más capaces para aprender e influir en las instituciones públicas, pero las mujeres tradicionalmente han tenido un menor número de años educativos (Dassonneville \& McAllister, 2018; Fraile \& Gómez, 2017; Hernández y Marañón, 2017). Asimismo, la discusión política promueve mayor conocimiento político, pero las mujeres han sido socializadas para evadir las conversaciones políticas y apartarse de funciones públicas (Ferrín et al., 2019; Fox \& Lawless, 2004; Wolak \& McDevitt, 2011). El estado socioeconómico es otro aspecto de importancia, pues, a mayor nivel económico, mayor conocimiento, pero las carreras femeninas se concentran en las ramas peor pagadas (Dryler, 1998; Revesz, 2017).

En el caso mexicano, se aúnan, además, características específicas. Por ejemplo, durante los años de socialización política, las niñas mexicanas de 12 a 19 años dedican un mayor número de horas a cuidar del hogar y a sus familiares (Instituto Nacional de las Mujeres [Inmujeres], 2017) y se inscriben a carreras tradicionalmente más femeninas y peor pagadas (Zamarrón, 2017). Estas prácticas terminan fomentando y reforzando desigualdades estructurales, como mayor ingreso masculino, mayor representación política y puestos de liderazgo para los hombres (Inmujeres, 2015).

De tal forma, a partir del concepto de información política (Delli \& Keeter, 1996), como resultado de la socialización política (Nateras, 2003; Neundorf \& Smets, 2020; Sapiro, 2004; Torney-Purta et al., 2007), y las diferencias de género (Bigler et al., 2013; Dryler, 1998; Fraile \& Sánchez- 
Vítores, 2019; Leaper, 2014; Raffaelli \& Ontai, 2004), las siguientes hipótesis y preguntas de investigación son propuestas:

- H1: Los factores de socialización política serán diferentes entre niños y niñas.

- $\quad H 2$ : El conocimiento político de los niños será mayor que él de las niñas.

- PI1: ¿Cómo influye la socialización política entre los niños y las niñas del estado de Nuevo León en su conocimiento político? ¿Qué diferencias particulares existen para las niñas?

- Además, dado el potencial de las redes sociales para actuar como mediador del conocimiento político (Cacciatore et al., 2018; Gil de Zúñiga et al., 2017; Lee \& Xenos, 2019; Prior, 2005), se propone la siguiente pregunta de investigación:

- PI2: ¿Cómo influye la socialización política al conocimiento político de los niños en el estado de Nuevo León (México) en consideración al uso de las redes sociales?

\section{Método}

Para responder a las preguntas de investigación e hipótesis, este estudio hace uso de la encuesta formación cívica y socialización política en la educación básica, efectuada entre abril y mayo 2017 a alumnos de quinto año (10-11 años) y sexto año (12-13 años) de primaria en el estado de Nuevo León (México). Aun cuando la investigación sobre la socialización política en este segmento de la población es escasa (Huerta y García, 2008; López, 2003, Tapia 2003), el proceso de socialización política infantil tiene antecedentes en la década de los setenta. En uno de los primeros esfuerzos, Segovia (1975) emplea encuestas para determinar las actitudes políticas de los niños mexicanos. Tomándolo como fuente, este proyecto diseñó un guion para realizar grupos focales y de ahí rediseñar escalas previamente usadas, y así dar origen a nuestro cuestionario. Por ejemplo, de estos grupos se evidencia el cambio generacional en el uso de dispositivos digitales para informarse y se incluyen preguntas detalladas sobre el consumo y contenido de redes sociales. Igualmente, la mayoría de las publicaciones sobre conocimiento político se han basado en el modelo (mo- 
tivación-habilidad-oportunidad) establecido por Delli \& Keeter (1996). A través de encuestas y experimentos, los autores y análisis subsecuentes (véase sección sobre brecha de género e información política) han examinado el modelo en su totalidad o se han enfocado en aspectos específicos. En este caso, exploramos el conocimiento político como resultado del modelo en conjunto, pero discriminando sobre el tipo de información consumida y el género.

La encuesta, mediante un muestreo probabilístico por conglomerados, seleccionó una muestra representativa de alumnos $(\mathrm{n}=1539)$ de escuelas de la zona metropolitana $(56,4 \%)$ y el resto de las zonas $(19,12 \%)$ del estado de Nuevo León. En dicho estado, hay 2569 centros que imparten educación primaria y se seleccionaron al azar 53 institutos (48 públicos y 5 privados), de los cuales 32 están en la zona metropolitana y 21 en otras zonas. Por tratarse de niños, la forma en la que se aplicó la encuesta fue mediante autorización previa por parte de la institución educativa y de los padres. Los encuestadores debían presentarse en la escuela plenamente identificados para acceder a los niños encuestados y solo en el grupo asignado. Para los propósitos de este estudio, se utilizan las preguntas referentes a conocimiento político, socialización política, eficacia interna y externa, consumo de medios, y preguntas sobre datos demográficos como control.

Para medir la socialización política, se hace uso de las preguntas sobre las rutinas familiares y escolares:

- Familia: Se considera la educación de la madre $(\mathrm{M}=2,97, \mathrm{SD}=1,26)$, del padre $(M=3,03, S D=1,26)$ y si el lugar donde escuchan más sobre política es la casa (17\%), medida de 0 (sin estudios) a 6 (doctorado).

- Escuela: Se utiliza el índice de formación cívico-político (en la escuela estoy expuesto a mecanismos participativos, mis profesores promueven valores democráticos, los alumnos tenemos un órgano representativo) $(M=3,56, S D=0,86, C$-alpha $=0,77)$ y si el lugar donde escuchan más de política es la escuela (39\%), medidas de 0 (nada) a 5 (bastante) (Imhoff \& Brussino, 2013; Stonjic, 2015). 
- $\quad$ Familia y escuela: Se hace uso de la escala de conversación política interpersonal (qué tanto hablan con ellos de política sus familiares y maestros $)(M=2,30, S D=0,79, C$-alpha $=0,81)$ y el entorno de conversación política (qué tanto hablan de política sus familiares y maestros $)(M=2,52, S D=0,67, C$-alpha $=0,72)$. Los índices son 0 (nada) a 5 (bastante).

El conocimiento político se construye a través de un índice conformado por las respuestas correctas a 18 preguntas sobre figuras políticas, instituciones y procedimientos electorales como número de estados en México, poderes del Estado, duración del mandato presidencial, nombre del presidente de México, del gobernador de Nuevo León, año electoral presidencial, así como identificación de varias personalidades políticas $(M=10,42$, $\mathrm{SD}=3,61$ ) (De Vresse, 2005; Rojas, 2006). Se recodificaron como "no sé" las repuestas ausentes.

Además, se controla por variables que miden la motivación-habilidad-oportunidad $(0-$ nada,- 5 -bastante- $)$ :

- Motivación: Interés en la política $(\mathrm{M}=2,99, \mathrm{SD}=1,02)$.

- Habilidad: Se midió a través del sentimiento político de eficacia interna (votar es útil, deseo de involucrarse en la política al ser grande, deseo de ser presidente o gobernante, deseo de unirse con otros para mejorar el país, reconocimiento del derecho de niños a participar en la política y de la importancia de los niños para la política, y si la política es difícil de entender $(\mathrm{M}=3,61, \mathrm{SD}=0,74, \mathrm{C}$-alpha $=0,65) \mathrm{Y}$ a través del sentimiento político de eficacia externa (como niño tengo derechos, mis papás y maestros me permiten exponer mis ideas, los maestros deben escuchar a sus alumnos, mis papás tienen en cuenta mi opinión $(M=4,41, S D=0,64, C$-alpha = 0,76) (De Vresse, 2005; Rojas, 2006).

- $\quad$ Oportunidad: Se midió por la atención a política por medios tradicionales (qué tanto se enteraban de política a través de noticias de política internacional, nacional, local o programas de política tradicionales $(\mathrm{M}=2,89, \mathrm{SD}=0,96, \mathrm{C}$-alpha $=0,88)$, los medios socia- 
les (adquisición de noticias que navegan por webs de política, redes sociales, $\mathrm{M}=2,81, \mathrm{SD}=1,10$, C-alpha $=0,71)$ y el consumo diario de horas de redes sociales (Facebook, Twitter, etc., $\mathrm{M}=1,30 \mathrm{~h}$, $\mathrm{SD}=1,02$, C-alpha $=0,84$ ). En general, los niños entrevistados dedicaron en promedio $2,86 \mathrm{~h}$ diarias a navegar por internet, con solo el $5 \%$ de la muestra no utilizándolo (cero horas) y el $91 \%$ dedicando entre menos de 1 h y más de $6 \mathrm{~h}$ diarias. El principal propósito declarado fue buscar ocio (Google, Yahoo!, etc.) (60\%). En referencia a las redes sociales, solo un $3 \%$ declararon no dedicar nada de tiempo a las redes sociales.

Para responder $\mathrm{H} 1$, las preguntas de socialización política entre niñas y niños fueron analizadas en conjunto y después comparadas individualmente entre ambos grupos en una prueba de la t de Student. Para analizar el conocimiento político entre ambos grupos $(\mathrm{H} 2)$, se obtuvo la media de las preguntas individuales y el índice de conocimiento en general, y fueron comparadas entre hombres y mujeres vía prueba de chi cuadrada y de la t de Student. Para responder a la PI1 sobre la influencia de la socialización política en el conocimiento político, se realizó un análisis multivariado con el índice de conocimiento como variable dependiente y los indicadores de socialización política como determinantes, con las variables motivación-habilidad-oportunidad-factores estructurales como herramientas de control. Finalmente, para responder a la PI2 sobre la influencia de la socialización política en el conocimiento político mediante las redes sociales, se condujo un par de análisis de mediación entre la socialización política (conversación interpersonal y entorno político) ${ }^{2}$ y el conocimiento político, con el uso de redes sociales como mediador. Se utilizó el modelo 4 (con género y edad como covariantes) del paquete macro PROCESS del programa SPSS.

\section{Resultados}

El número total de niños que participaron en la encuesta fue de 1539, pero solo 1389 niños y niñas identificaron claramente su género, por lo que se procedió a invalidar los cuestionarios de los niños que contestaron en las dos opciones provistas cuando su elección no era legible o si la opción no 
fue contestada. Los niños representaron el $48 \%(\mathrm{n}=662)$ de la muestra y las niñas el $52 \%(\mathrm{n}=727)$. Su edad media fue de 11,87 años. En general, el índice de conocimiento político, nuestra variable dependiente, tiene un promedio de $M=10,43$ respuestas correctas sobre 18 reactivos $(S D=3,61)$.

Para responder si los factores de socialización política son diferentes entre niños y niñas ( $\mathrm{H} 1)$, se compararon las variables de socialización política. Los resultados indican una similitud de respuestas entre ambos géneros y señalan que la socialización en familia a esta edad no difiere mucho entre ambos grupos. La educación de los padres $\left(\mathrm{M}_{\text {madre }}=2,97\right.$ y $\left.\mathrm{M}_{\text {padre }}=3,03\right)$, el entorno de la conversación política $(\mathrm{M}=2,52, \mathrm{SD}=0,66)$, la conversación interpersonal $(M=2,52, S D=0,78)$ y los lugares donde aprenden sobre ella (en casa $=17,2 \%$ y escuela $=39 \%$ ) no son estadísticamente diferentes, excepto por la formación cívico-política, en la que las niñas parecen beneficiarse más $\left(\mathrm{M}_{\text {ninas }}=3,60\right.$ y $\left.\mathrm{M}_{\text {niños }}=3,50\right), \mathrm{t}(1,282)=1,77, \mathrm{p}=0,04$. Estos resultados rechazan parcialmente $\mathrm{H} 1$. La socialización familiar es similar entre niños y niñas, pero la socialización en la escuela difiere.

Para responder la $\mathrm{H} 2$, se compararon las medias del conocimiento político entre niños y niñas, y las proporciones de las respuestas a las preguntas individuales. Los resultados indicaron que el conocimiento político de las niñas $(M=10,38, S D=3,61)$ es marginalmente inferior al de los niños $(\mathrm{M}=10,72, \mathrm{SD}=3,51), \mathrm{t}(1375)=-1,78, \mathrm{p}=0,08)$, aunque no significativamente, lo que rechaza $\mathrm{H} 2$. A pesar de estos resultados, hubo un par de patrones interesantes. Por ejemplo, los niños respondieron correctamente en mayor proporción a preguntas sobre figuras nacionales (número de estados, conocimiento de Osorio Chong, Miguel Ángel Mancera y El Chapo), mientras que las niñas supieron representativamente más acerca de figuras femeninas, locales (conocimiento de el Bronco, Margarita Zavala) y no políticas (¿Carlos Slim es político?). De igual manera, el número de respuestas ausentes o "no sé" fue estadísticamente mayor en las niñas. Los otros determinantes (motivación-habilidad-oportunidad) son estadísticamente similares, excepto por el sentimiento de eficacia política externa y el consumo de redes sociales, en el que es significativamente superior en las niñas $\left(M_{\text {niñas }}=4,49, S D=0,58\right.$ y $M_{\text {niñas }}=1,42, S D=1,09$, respectivamente $)$. 
Para responder si la socialización política influye en el conocimiento político (PI1), se realizó primero un análisis multivariado para predecir el índice de conocimiento general con los indicadores de socialización política como determinantes, controlando variables tradicionales del conocimiento político como motivación-habilidad-oportunidad y factores estructurales. Los resultados de la regresión múltiple indicaron que estas variables explicaron un $13 \%$ de la variación en sofisticación política $\left(\mathrm{R}^{2}=0,13, \mathrm{~F}\right.$ $(15508)=5,18, \mathrm{p}<0,001)$. La tabla 1 enlista todos los determinantes $\mathrm{y}$ su poder predictivo. De la tabla se puede observar que ser niño predice un aumento en el índice de información política $(\beta=0,08, p=0,05)$, cuando todos los demás elementos son considerados y constantes. Los elementos socializantes que predicen significativamente el incremento de conocimiento político son los grados de estudio de la madre (aunque marginalmente $(\beta=0,10, p=0,08)$, el entorno de conversación política $(\beta=0,14$, $\mathrm{p}=0,06)$ y la reducción de la conversación política interpersonal $(\beta=-0,23$, $\mathrm{p}<0,001)$. La formación político-cívica no tuvo un incremento en la sofisticación política.

Para comparar cuáles son los determinantes en específico del conocimiento político para las niñas, se realizó un análisis multivariado por género. La tabla 1 incluye también los principales determinantes por separado. Los resultados indican que el modelo de conocimiento político para las niñas explica un $15 \%$ de la variación en la sofisticación política $\left(\mathrm{R}^{2}=\right.$ $0,15, \mathrm{~F}(14251)=3,22$, $\mathrm{p}<0,001)$, mientras que el modelo de sofisticación política para los niños explica un $17 \%$ de la variación $\left(\mathrm{R}^{2}=0,17, \mathrm{~F}\right.$ $(14243)=3,42, \mathrm{p}<0,001)$. Referente a las variables socializantes, se pueden observar ciertas diferencias. Por ejemplo, el entorno de conversación política (qué tanto hablan de política sus familiares y maestros) estimula la sofisticación política en las niñas $(\beta=0,25, \mathrm{p}=0,02)$, mas no así la conversación política interpersonal (qué tanto hablan con ellas de política sus familiares y maestros) $(\beta=-0,36, p<0,001)$. Los medios afectaron el conocimiento político de manera negativa a las niñas. Por ejemplo, la atención a política por medios digitales y el consumo de redes sociales redujo significativamente su nivel de información política. 


\section{Tabla 1. Determinantes del conocimiento político general en niños y niñas}

\begin{tabular}{|c|c|c|c|c|c|c|c|c|c|}
\hline & \multicolumn{3}{|c|}{ General } & \multicolumn{3}{|c|}{ Niñas } & \multicolumn{3}{|c|}{ Niños } \\
\hline & B & EE & $\beta$ & B & EE & $\beta$ & B & EE & $\beta$ \\
\hline (Constante) & 2,34 & 2,41 & & 7,88 & 3,84 & & $-1,6$ & 2,99 & \\
\hline \multicolumn{10}{|l|}{ Factores estructurales } \\
\hline Género $(1=\mathrm{F}, 2=\mathrm{H})$ & 0,53 & 0,27 & $0,08^{*}$ & & & & & & \\
\hline Edad & 0,27 & 0,19 & 0,06 & $-0,2$ & 0,29 & $-0,04$ & 0,71 & 0,25 & $0,2^{* *}$ \\
\hline \multicolumn{10}{|l|}{ Socialización política } \\
\hline Estudios de la madre & 0,24 & 0,14 & $0,10^{\mathrm{a}}$ & 0,24 & 0,20 & 0,09 & 0,27 & 0,18 & 0,11 \\
\hline Estudios del padre & 0,16 & 0,14 & 0,06 & 0,09 & 0,21 & 0,04 & 0,21 & 0,19 & 0,09 \\
\hline $\begin{array}{l}\text { Conversación política } \\
\text { interpersonal }\end{array}$ & $-1,00$ & 0,33 & $-0,2^{* *}$ & $-1,7$ & 0,53 & $-0,4^{* *}$ & $-0,5$ & 0,41 & $-0,12$ \\
\hline $\begin{array}{l}\text { Entorno con conversación } \\
\text { política }\end{array}$ & 0,73 & 0,39 & $0,14^{\mathrm{a}}$ & 1,39 & 0,61 & $0,25^{*}$ & 0,14 & 0,51 & 0,03 \\
\hline $\begin{array}{l}\text { Lugar de conversación política } \\
\text { (casa) }\end{array}$ & $-0,25$ & 0,38 & $-0,03$ & $-0,3$ & 0,56 & $-0,03$ & $-0,1$ & 0,52 & $-0,02$ \\
\hline $\begin{array}{l}\text { Lugar de conversación política } \\
\text { (escuela) }\end{array}$ & $-0,14$ & 0,29 & $-0,02$ & $-0,2$ & 0,44 & $-0,03$ & 0,07 & 0,39 & 0,01 \\
\hline Formación cívico-política & $-0,17$ & 0,18 & $-0,04$ & $-0,2$ & 0,26 & $-0,05$ & $-0,2$ & 0,24 & $-0,05$ \\
\hline \multicolumn{10}{|l|}{ Motivación } \\
\hline Interés en la política & 0,35 & 0,16 & $0,11^{*}$ & 0,31 & 0,26 & 0,08 & 0,34 & 0,21 & 0,12 \\
\hline \multicolumn{10}{|l|}{ Habilidad } \\
\hline $\begin{array}{l}\text { Sentimiento de eficacia política } \\
\text { interna }\end{array}$ & $-0,51$ & 0,21 & $-0,1^{*}$ & $-0,4$ & 0,32 & $-0,09$ & $-0,6$ & 0,28 & $-0,2^{*}$ \\
\hline $\begin{array}{l}\text { Sentimiento de eficacia política } \\
\text { externa }\end{array}$ & 1,24 & 0,24 & $0,2^{* *}$ & 1,26 & 0,41 & $0,2^{* *}$ & 1,25 & 0,30 & $0,3^{* *}$ \\
\hline \multicolumn{10}{|l|}{ Oportunidad } \\
\hline $\begin{array}{l}\text { Atención a política por medios } \\
\text { tradicionales }\end{array}$ & 0,51 & 0,19 & $0,15^{*}$ & 0,78 & 0,28 & $0,2^{* *}$ & 0,24 & 0,25 & 0,08 \\
\hline $\begin{array}{l}\text { Atención a política por redes } \\
\text { sociales }\end{array}$ & $-0,24$ & 0,15 & $-0,09$ & $-0,6$ & 0,23 & $-0,2^{* *}$ & 0,16 & 0,21 & 0,06 \\
\hline Consumo de redes sociales & $-0,40$ & 0,14 & $-0,1^{* *}$ & $-0,4$ & 0,21 & $-0,12^{*}$ & $-0,4$ & 0,19 & $-0,1^{*}$ \\
\hline $\mathrm{R}^{2}$ & 0,13 & & & 0,15 & & & 0,17 & & \\
\hline $\begin{array}{l}\text { B: Coeficiente beta no estandari } \\
\text { Nota: }{ }^{a}=p \leq 0,10,{ }^{*}=p \leq 0,05,{ }^{* *}\end{array}$ & EE: er & ípic & & & & & & & \\
\hline
\end{tabular}

Fuente: elaboración propia. 
Además de visualizar el impacto de la socialización política al conocimiento político mediante el uso de redes sociales (PI2), se realizaron dos análisis de mediación. El primero exploró el consumo de redes sociales como mediador entre los dos elementos significativos de la socialización política y el conocimiento político de los niños: la conversación interpersonal y el entorno de la conversación política. Los resultados indicaron un efecto indirecto de la conversación interpersonal en el conocimiento político a través del consumo de las redes sociales, $\beta=-0,0303, S E=0,02$ [IC $95 \%$ : $(-0,0712,-0,0034)]$, en el que la influencia de la conversación interpersonal en el conocimiento político fue mediada negativamente por el uso de las redes sociales.

De manera semejante, el segundo análisis exploró el consumo de redes sociales como mediador entre el entorno de conversación política (qué tanto hablan de política a su alrededor) y el conocimiento político. Los resultados indicaron un efecto indirecto del entorno político en el conocimiento político a través del consumo de las redes sociales, $\beta=-0,04$, $S E=0,02[$ IC $95 \%:(-0,0808,-0,0046)]$. En sí, el efecto del entorno de conversación política en el conocimiento político estuvo mediado negativamente por el uso de las redes sociales. La tabla 2 resume ambas mediaciones. La siguiente sección desarrolla las implicaciones y limitaciones de la información presentada.

\section{Tabla 2. Efectos directos e indirectos de la socialización política en el conocimiento de niños y niñas}

\begin{tabular}{|l|c|c|c|c|c|c|}
\hline & $\mathbf{X}-\mathbf{M}$ & $\mathbf{M}-\mathbf{Y}$ & $\begin{array}{c}\text { Directo } \\
\mathbf{X}-\mathbf{Y}\end{array}$ & $\begin{array}{c}\text { Indirecto X-Y } \\
\text { (mediación) }\end{array}$ & $\begin{array}{c}\text { Género } \\
\mathbf{M}-\mathbf{Y}\end{array}$ & $\begin{array}{c}\text { Edad } \\
\mathbf{M}-\mathbf{Y}\end{array}$ \\
\hline Conversación interpersonal política & $0,13^{* *}$ & $-0,23^{*}$ & $-0,27^{*}$ & $-0,03^{*}$ & $0,40^{* *}$ & 0,32 \\
\hline Entorno conversación política & $0,13^{* *}$ & $-0,26^{* *}$ & 0,09 & $-0,04^{*}$ & $0,39^{\mathrm{a}}$ & $0,42^{* *}$ \\
\hline $\begin{array}{l}\text { Nota: }{ }^{2}=\mathrm{p} \leq 0,10,{ }^{*} \mathrm{p}<0,05,{ }^{* *} \mathrm{p}<0,001 . \text { Edad y género son covariantes en ambos modelos. El mediador es el consumo } \\
\text { de redes sociales. }\end{array}$
\end{tabular}

Fuente: elaboración propia. 


\section{Conclusiones}

El objetivo de este trabajo fue analizar los potenciales factores que afectan la brecha de género en el conocimiento político de niños y niñas, a partir de la literatura propuesta por los determinantes de la sofisticación política, las redes sociales y la puntualización de la socialización política y de género. Para lograrlo, se utilizó la encuesta formación cívica y socialización política en la educación básica en el estado de Nuevo León (México) realizada en 2017. Aunque múltiples estudios sobre la brecha de género en el mundo han reafirmado los distintos niveles de información que tienen hombres y mujeres, nuestra contribución reside en un análisis en los años formativos de los futuros ciudadanos, el contexto mexicano y la inclusión de nuevas variables que previamente habían sido consideradas como estables (acceso y consumo de información).

La comparación de las variables de socialización política entre los niños y las niñas encuestados indicó que estas son bastante equivalentes en ambos géneros (H1), excepto la formación cívico-política, la eficacia política externa y el consumo de redes sociales, que es mayor en las niñas. Es interesante que a esta edad ( $\mathrm{M}=11,87$ años) la eficacia política externa es mayor en las niñas cuando a nivel nacional es superior en los hombres (García, 2015; Hernández y Marañón, 2017). También resalta que las ninas encuestadas consumen redes sociales significativamente en mayor medida que los niños.

Dado que existe una brecha significativa de conocimiento político en la política entre hombres y mujeres a nivel nacional y mundial, se asumió lo mismo para los niños encuestados del estado de Nuevo León, sin embargo, esta hipótesis fue rechazada $(\mathrm{H} 2)$. El conocimiento político de los niños es solo marginalmente superior al de las niñas, pero no estadísticamente representativo. Sin embargo, cuando se estudiaron las respuestas individualmente, se observó que las niñas dejaban preguntas sin responder en mayor manera, su forma de manifestar "no sé", lo cual es parecido a lo que otros estudios han encontrado (Mondak \& Creel, 2001). También se advirtió que los niños tenían mayor conocimiento de figuras y datos nacionales (p. ej., 
estados en la República, políticos nacionales), mientras que las niñas tenían mayor conocimiento de figuras locales (p. ej., gobernador del estado) y femeninas (p. ej., Carmen Aristegui, periodista, y Margarita Zavala, precandidata presidencial). Estas diferencias en el conocimiento muestran patrones que se han encontrado en otros países. Por ejemplo, Dolan (2011) encontró que el tipo de conocimiento femenino está más relacionado con la comunidad (p. ej., actores locales) y con figuras con las que se relacionan (p. ej., líderes mujeres).

Para analizar cómo estos elementos socializantes afectan el conocimiento político y la influencia en particular hacia las niñas (PI1), se efectuó un modelo general, un modelo de conocimiento político para las niñas y otro para los niños. Esto permitió trazar los determinantes generales y cuáles son específicos a las características de cada género. El primer modelo confirma el modelo tradicional (Delli \& Keeter, 1996), con las variables motivación-habilidad-oportunidad, de modo que son estadísticamente significativas. Aunque no hay diferencias significativas en el conocimiento político entre niños y niñas encuestados cuando se controlan otros predictores, se observó que ser niño anticipa mayor conocimiento político.

$\mathrm{Al}$ revisar los modelos individuales de conocimiento político para niños y niñas, se encontró que para las niñas de la encuesta la información política no proviene de un interés propio o de un sentimiento de eficacia interna (p. ej., querer ser presidente, encontrar la política "fácil”, etc.), sino que ellas aprenden más de política en un entorno de conversación política y de eficacia externa (p. ej., cuando los adultos en su vida platican de política y consideran sus opiniones), aunque no dirigido a ellas en específico (cuando sus familiares y maestros hablan directamente con ellas tenía un efecto negativo). Los medios tradicionales les ayudan a aprender, mas no así las redes sociales. A esta edad, las niñas parecen absorber la información a su alrededor cuando no son el centro de atención (Wolak \& McDevitt, 2011). Una cualidad diferente del aprendizaje de conocimiento político en los niños que ocurre más naturalmente con la edad. Los niños parecen recibir los beneficios de la educación en cuestiones políticas, sin embargo, la edad no ayuda a las niñas a adquirir mayor conocimiento político. Los 
futuros estudios deberían profundizar con entrevistas qué sucede en estos años, pues los resultados indican que este es el momento en que la brecha de género empieza a manifestarse.

Referente a la influencia de las redes sociales, los resultados de este estudio coinciden con estudios pasados en el efecto negativo de las redes sociales (Cacciatore et al., 2018; Gil de Zúñiga et al., 2017; Kim et al., 2013; Lee \& Xenos, 2019). Si bien internet permite la adquisición de información de manera más eficaz (Gil de Zúñiga et al., 2017; Prior, 2005), el alto consumo de redes sociales interviene de manera negativa en el conocimiento político y en aspectos críticos de la socialización política como conductor del conocimiento político. Por ejemplo, en nuestro estudio, tanto la conversación interpersonal (hablar con los niños de política) como el entorno de conversación política (que los niños escuchen a adultos hablar de política) fomentaron el mayor uso de redes sociales, solo para que estos a su vez disminuyeran el conocimiento político. En este contexto, es posible que después de conversaciones políticas con familiares y maestros, los niños acudieran a aclarar sus dudas a través de las redes sociales, únicamente para encontrar información discordante o ser distraídos por el entretenimiento que suponen. Los estudios subsiguientes podrían investigar cuáles son los mecanismos específicos por los que la socialización política promueve el deseo de adquirir información, solo para ver su efecto eliminado por el internet (esto es, acceso a noticias falsas). Especialmente para las niñas, pues su consumo es mayor del de los niños.

En resumen, estos resultados extienden la capacidad predictiva del modelo teórico generado por Delli \& Ketter (1996) en tres formas. Primero, contribuye a la investigación sobre el conocimiento político en países latinoamericanos, puesto que la mayoría de los estudios continúa concentrándose en contextos estadounidenses y europeos. Segundo, reconoce la repercusión de los años formativos y responde a los llamados de indagar en qué momento la brecha de género se vuelve un detrimento para la ciudadanía (Ferrín et al., 2014; Fraile \& Sánchez-Vítores, 2019). Tercero, la disponibilidad de información se considera una variable constante en la mayoría de las publicaciones sobre sofisticación política, por lo que aquí se analiza 
el impacto de factores vigentes como el uso de las redes sociales y su efecto no solo en el conocimiento político, sino también en la brecha de género. Si bien los resultados no ratifican la desigualdad de conocimiento político que se observa en el mundo en las mujeres (Delli \& Keeter, 1996; Jerit et al., 2006; Luskin, 1990), sí deja entrever los indicios de donde proviene. En particular, el hecho de que las mujeres tuvieran un proceso de aprendizaje diferente de los hombres (p. ej., conversación política interpersonal y entorno con conversación política) y diferente consumo de redes sociales da crédito a los efectos de la socialización de género (Bigler et al., 2013; Dryler, 1998; Ferrín et al., 2014; Leaper, 2014; Raffaelli \& Ontai, 2004). Los futuros estudios podrían considerar un estudio longitudinal y verificar en qué años la brecha de género se convierte en estadísticamente representativa y por qué la atención y el consumo de política mediante redes sociales es diferente para niños y niñas.

Por otra parte, los lectores deben considerar ciertas limitaciones en este proyecto. Primero, los modelos ayudan a explicar los determinantes de conocimiento político, pero su poder predictivo es bajo. Es probable que la predicción sea baja para alumnos que comparten características demográficas, educacionales y socioeconómicas similares. Segundo, este estudio basa las inferencias hechas en la socialización política de las mujeres en la bibliografía de socialización de género (Bigler et al., 2013; Dryler, 1998; Leaper, 2014; Ferrín et al., 2014; Raffaelli \& Ontai, 2004; Wolak \& McDevitt, 2011), pero no hubo variables diseñadas exclusivamente para esta función. Los estudios consecutivos se beneficiarían de incluir variables con una perspectiva de género, por ejemplo, si el contenido de las conversaciones políticas de padres a niños difiere según el sexo, si existen diferentes conceptos de conocimiento político entre niños y niñas, o entrevistas con padres/maestros acerca de sus expectativas políticas para niños y niñas. Por último, la educación es uno de los factores más determinantes del conocimiento político y nuestro estudio incluye solo a niños que están en la escuela. Si bien ayuda a estandarizar los resultados, dificulta la inferencia de conocimiento político en adultos, puesto que muchos ciudadanos en México no han tenido una educación formal. 
Sin descartar estas limitaciones, este estudio sugiere que a la temprana edad de 10 a 11 años ya pueden distinguirse los vestigios de la brecha en conocimiento político entre niños y niñas, que marca y deteriora la vida política de las mujeres en su vida adulta. En México, las mujeres son vehementes participantes electorales, pero su voto es desinformado y con pocas repercusiones para su vida diaria (García, 2015; Soto et al., 2014). El conocimiento político está ligado al compromiso político y al uso práctico de derechos ciudadanos en la vida diaria, pero también al reconocimiento de desigualdades políticas (Dassonneville \& McAllister, 2018; Fraile \& Sánchez-Vítores, 2019). De estos resultados podemos esperar que el escrutinio político se extienda también a otros aspectos de las vidas de las mujeres, como el reconocimiento de la desigualdad en representación política, la disparidad de pago y la violencia que viven diariamente niñas y mujeres. De este estudio se puede observar que las niñas tienen tanta eficacia interna e interés en la política como sus compañeros, pero cuando llegan a su vida adulta activamente evaden y toman una posición pasiva en las conversaciones políticas (Hernández y Marañón, 2017). Igualdad de acceso a la escuela, politización en la familia y acceso a medios de calidad son los principales socializantes de un conocimiento político saludable y eficaz, pero, como este y otros estudios han señalado, no es suficiente. De forma práctica, se podría involucrar y motivar las conversaciones políticas en las que las niñas estén presentes, incluso si no participan activamente todo el tiempo, la enseñanza cívica con un enfoque de género que reconozca su aprendizaje característico y las motive a la participación política futura, y el uso crítico de las redes sociales (p. ej., reconocimiento de noticias falsas, pero también considerando el consumo específico de redes sociales que realizan). Sus consecuencias permitirán empoderar a las niñas en su día a día y en su proceso de formación ciudadano, en ruta a convertirse en un miembro versado no solo en los eventos y las figuras políticas que las rodean, sino en un agente activo del sistema político-social. 


\section{Referencias}

Abendschön, S. \& Tausendpfund, M. (2017). Political knowledge of children and the role of sociostructural factors. American Behavioral Scientist, 61(2), 204-221. https://doi.org/10.1177/0002764216689122

Assis, C. de. (2019, junio 24). Crece uso de Instagram y WhatsApp para el consumo de noticias en línea en Argentina, Brasil, Chile y México, señala informe. https://latamjournalismreview.org/es/articles/ crece-uso-de-instagram-y-whatsapp-para-el-consumo-de-noticias-en-linea-en-argentina-brasil-chile-y-mexico-senala-informe/

Barbeito, R.-L. (2002). La familia y los procesos de socialización y reproducción sociopolíticas de juventud. Revista de Estudios de Juventud, 58(2), 1-11.http://www.injuve.es/sites/default/files/articulo6.pdf

Batista Pereira, F. (2012). Gender and Community Participation in Latin America and the Caribbean. Americas Barometer Insights 78. Latin American Public Opinion Project. Vanderbilt University. https://www. vanderbilt.edu/lapop/insights/IO878en.pdf

Bigler, R., Roberson Hayes, A. \& Hamilton, V. (2013). The role of schools in the early socialization of gender differences. En Encyclopedia on early childhood development. http://www.child-encyclopedia.com/ gender-early-socialization/according-experts/role-schools-earlysocialization-gender-differences

Bode, L. (2016). Political news in the news feed: Learning politics from social media. Mass Communication and Society, 19(1), 24-48. https:// doi.org/10.1080/15205436.2015.1045149

Bode, L. \& Vraga, E. K. (2015). In related news, that was wrong: The correction of misinformation through related stories functionality in social media. Journal of Communication, 65(4), 619-638. https:// doi.org/10.1111/jcom.12166 
Cacciatore, M. A., Yeo, S. K., Scheufele, D. A., Xenos, M. A., Brossard, D. \& Corley, E. A. (2018). Is Facebook making us dumber? Exploring social media use as a predictor of political knowledge. Journalism \& Mass Communication Quarterly, 95(2), 404-424. https://doi. org/10.1177/1077699018770447

Dassonneville, R. \& McAllister, I. (2018). Gender, political knowledge, and descriptive representation: The impact of long-term socialization. American Journal of Political Science, 62(2), 249-265. https://doi. org/10.1111/ajps.12353

Delli Carpini, M. \& Keeter, S. (1996). What Americans know about politics and why it matters. Yale University Press.

Dolan, K. (2011). Do women and men know different things? Measuring gender differences in political knowledge. The Journal of Politics, 73(1), 97-107. https://doi.org/10.1017/S0022381610000897

Dow, J. K. (2009). Gender differences in political knowledge: Distinguishing characteristics-based and returns-based differences. Political Behavior, 31(1), 117-136. https://doi.org/10.1007/s11109-008-9059-8

Dryler, H. (1998). Parental role models, gender and educational choice. British Journal of Sociology, 49(3), 375-398. https://doi. org/10.2307/591389

Encuesta Nacional sobre Cultura Política y Prácticas Ciudadanas (Encup) 2012. http://bdsocial.inmujeres.gob.mx/index.php/encup-28/ encuesta-nacional-sobre-cultura-politica-y-practicas-ciudadanas/17-acervo/acervo/270-encuesta-nacional-sobre-culturapolitica-y-practicas-ciudadanas-encup-2012

Ferrín, M., Fraile, M. \& García-Albacete, G. M. (2019). Adult roles and the gender gap in political knowledge: A comparative study. West European Politics, 42(7), 1368-1389. https://doi.org/10.1080/014 02382.2019.1577069 
Fondo de las Naciones Unidas para la Infancia. (2014). Observaciones Generales del Comité de los Derechos del Niño. https://www.unicef. org/UNICEF-ObservacionesGeneralesDelComiteDeLosDerechosDelNino-WEB.pdf

Fox, R. L. \& Lawless, J. L. (2004). Entering the arena? Gender and the decision to run for office. American Journal of Political Science, 48(2), 264-280. https://doi.org/10.1111/j.0092-5853.2004.00069.x

Fraile, M. (2014). Do women know less about politics than men? The gender gap in political knowledge in Europe. Social Politics, 21(2), 261289. https://doi.org/10.1093/sp/jxu006

Fraile, M. \& Gómez, R. (2017). Why does Alejandro know more about politics than Catalina? Explaining the Latin American gender gap in political knowledge. British Journal of Political Science, 47(1), 91112. https://doi.org/10.1017/S0007123414000532

Fraile, M. \& Sánchez-Vítores, I. (2020). Tracing the gender gap in political interest over the life span: A panel analysis. Political Psychology, 41(1), 89-106. https://doi.org/10.1111/pops.12600

García Escribano, J. J. y Frutos Balibrea, L. (1999). Mujeres, hombres y participación política: buscando las diferencias. Reis: Revista Española de Investigaciones Sociológicas, 86, 307-329. https://www.jstor. org/stable/40184154

García Trejo, Y. A. (2015). Gender differences in political knowledge: The case of Mexico (Tesis doctoral, University of Connecticut). https:// core.ac.uk/download/pdf/302390597.pdf

Gil de Zúñiga, H., Weeks, B. \& Ardèvol-Abreu, A. (2017). Effects of the news-finds-me perception in communication: Social media use implications for news seeking and learning about politics. Journal of Computer-Mediated Communication, 22(3), 105-123. https:// doi.org/10.1111/jcc4.12185 
Gülgönen, T. (2016). Participación infantil a nivel legal e institucional en México: ¿ciudadanos y ciudadanas? Revista Latinoamericana de Ciencias Sociales, Niñez y Juventud, 14(1), 81-93. http://dx.doi.or $\mathrm{g} / 10.11600 / 1692715 \mathrm{x} .1414081014$

Hernández, M. (2018). "Killed out of love”: A frame analysis of domestic violence coverage in Hong Kong. Violence Against Women, 24(12), 1454-1473. https://doi.org/10.1177/1077801217738581

Hernández Martínez, M. y Marañón, F. de J. (2017). Brecha de género en México: determinantes de la información política en las mujeres mexicanas. Revista Legislativa de Estudios Sociales y de Opinión Pública, 10(20), 65-99. https://dialnet.unirioja.es/servlet/ articulo? codigo $=6237326$

Huerta Wong, J. E. y García, E. (2008). La formación de los ciudadanos: el papel de la televisión y la comunicación humana en la socialización política. Comunicación y Sociedad, 10, 163-189. http://www.scielo.org. $\mathrm{mx} /$ scielo.php?script=sci_arttext\&pid=S0188-252X2008000200007

Hurtado, L.Á. (2019, junio 9). Mexicanos pasan en promedio entre 2 y 4 horas diarias en WhatsApp. https://www.dgcs.unam.mx/boletin/bdboletin/2019_408.html

Imhoff, D. \& Brussino, S. (2013). Participación sociopolítica infantil y procesos de socialización política: exploración con niños y niñas de la ciudad de Córdoba, Argentina. Liberabit, 19(2), 205213. http://www.scielo.org.pe/scielo.php?script=sci_arttext\&pi $\mathrm{d}=$ S1729-48272013000200006

Instituto Nacional de las Mujeres. (2015, diciembre 15). Desigualdad en cifras. Boletín, 1(8). http://cedoc.inmujeres.gob.mx/documentos_download/BoletinN8.pdf

Instituto Nacional de las Mujeres. (2017). Sistema de Indicadores de Género. http://estadistica.inmujeres.gob.mx/formas/index.php 
Jaime Castillo, A. M. (2000). Familia y socialización política: la transmisión de orientaciones ideológicas en el seno de la familia española. Reis: Revista Española de Investigaciones Sociológicas, 92, 71-92. https://dialnet.unirioja.es/servlet/articulo? codigo $=758098$

Jerit, J. \& Barabas, J. (2017). Revisiting the gender gap in political knowledge. Political Behavior, 39(4), 817-838. https://doi.org/10.1007/ s11109-016-9380-6

Jerit, J., Barabas, J. \& Bolsen, T. (2006). Citizens, knowledge, and the information environment. American Journal of Political Science, 50(2), 266-282. https://www.jstor.org/stable/3694272

Kim, Y., Chen, H. T. \& De Zúñiga, H. G. (2013). Stumbling upon news on the internet: Effects of incidental news exposure and relative entertainment use on political engagement. Computers in $\mathrm{Hu}$ man Behavior, 29(6), 2607-2614. https://doi.org/10.1016/j. chb.2013.06.005

Leaper, C. (2014). Gender: early socialization. Encyclopedia on early childhood development. http://www.child-encyclopedia.com/genderearly-socialization/according-experts/

Lee, S. \& Xenos, M. (2019). Social distraction? Social media use and political knowledge in two US Presidential elections. Computers in Human Behavior, 90, 18-25. https://doi.org/10.1016/j.chb.2018.08.006

Lizotte, M. K. \& Sidman, A. H. (2009). Explaining the gender gap in political knowledge. Politics \& Gender, 5(2), 127-151. https://doi. org/10.1017/S1743923X09000130

López Gómez, G. (2003). Televisión y socialización política de los niños en Monterrey y Juchitán (Tesis de maestría, Tecnológico de Monterrey). https://repositorio.tec.mx/bitstream/handle/11285/571255/ DocsTec_1489.pdf?sequence $=1$ 
Luskin, R. C. (1990). Explaining political sophistication. Political Behavior, 12(4), 331-361. https://doi.org/10.1007/BF00992793

Lyons, J. (2017). The family and partisan socialization in red and blue America. Political Psychology, 38(2), 297-312. https://doi.org/10.1111/ pops.12336

Mondak, J. J. \& Anderson, M. R. (2004). The knowledge gap: A reexamination of gender-based differences in political knowledge. The Journal of Politics, 66(2), 492-512. http://doi.org/10.1111/j.14682508.2004.00161.x

Mondak, J.J. \& Creel Davis, B. (2001). Asked and answered: Knowledge levels when we won't take 'don't know' for an answer. Political Behavior, 23(3), 199-224.https://doi.org/10.1023/A:1015015227594

Nateras Domínguez, J. O. (2003). Procesos de socialización política y construcción del pensamiento social en infantes y jóvenes: la ruta de la sociocognición. Polis: Investigación y Análisis Sociopolítico y Psicosocial,2(3), 49-78. https://www.redalyc.org/pdf/726/72620303.pdf

Neundorf,A. \& Smets, K. (2020). Political socialization and the making of citizens.https://doi.org/10.1093/oxfordhb/9780199935307.013.98

Pereira, M. F., Fraile, M. \& Rubal, M. (2015). Young and gapped? Political knowledge of girls and boys in Europe. Political Research Quarterly, 68(1), 63-76. https://doi.org/10.1177/1065912914554040

Prior, M. (2005). News vs. entertainment: How increasing media choice widens gaps in political knowledge and turnout. American Journal of Political Science, 49(3), 577-592.http://doi.org/10.2307/3647733

Raffaelli, M. \& Ontai, L. L. (2004). Gender socialization in Latino/a families: Results from two retrospective studies. Sex Roles, 50(5-6), 287299. https://doi.org/10.1023/B:SERS.0000018886.58945.06 
Revesz, R. (2017, octubre 17). Women around the world earn a quarter less than men, finds UN report. Independent. https://www.independent.co.uk/news/world/women-salaries-men-gender-paygap-world-un-report-a8005796.html

Rojas, H. (2006). Comunicación, participación y democracia. Universitas Humanística, 62, 109-142. http://www.scielo.org.co/ scielo.php?pid $=$ S0120-48072006000200006\&script $=$ sci arttext\&tlng=en

Romero, V. y Parás, P. (2013). Cultura política de la democracia en México y en las Américas, 2012: hacia la igualdad de oportunidades. Vanderbilt University. https://politica.itam.mx/sites/default/files/politicaitammx/noticias/aadjuntos/2013/09/countryreports2012_mexico_spanish_v18_central_lapop_7.22_final.pdf

Sapiro, V. (2004). Not your parents' political socialization: Introduction for a New Generation. Annual Review of Political Science, 7, 1-23. https://doi.org/10.1146/annurev.polisci.7.012003.104840

Segovia, R. (1975). La politización del niño mexicano. El Colegio de México.

Simon, A. (2017). How can we explain the gender gap in children's political knowledge? American Behavioral Scientist, 61(2), 222-237. https://doi.org/10.1177/0002764216689123

Soto Zazueta, I. M. y Cortez, W.W. (2014). Determinantes de la participación electoral en México. Estudios Sociológicos, 32(95), 323-353.https:// estudiossociologicos.colmex.mx/index.php/es/article/view/40

Stojnic Chávez, L. G. (2015). El efecto de la experiencia escolar en el desarrollo de actitudes favorables hacia la democracia como mejor sistema político: el caso de estudiantes peruanos recién graduados del nivel secundario. Colombia Internacional, 85, 111-139. http:// dx.doi.org/10.7440/colombiaint85.2015.04 
Tapia, E. (2003). Socialización política y educación cívica en los niños. Instituto Mora.

Torney-Purta, J., Barber, C. H. \& Wilkenfeld, B. (2007). Latino adolescents' civic development in the United States: Research results from the IEA Civic Education Study. Journal of Youth and Adolescence, 36(2), 111-125. https://doi.org/10.1007/s10964-006-9121-y

Trevor, M. C. (1999). Political socialization, party identification, and the gender gap. Public Opinion Quarterly, 63(1), 62-89. http://www. jstor.org/stable/2991269

Vreese, C. H. de. (2005). The spiral of cynicism reconsidered. European Journal of Communication, 20(3), 283-301. https://doi. org/10.1177/0267323105055259

Vreese, C. H. de. \& Elenbaas, M. (2008). Media in the game of politics: Effects of strategic metacoverage on political cynicism. The International Journal of Press/Politics, 13(3), 285-309. https://doi. org/10.1177/1940161208319650

Wolak, J. \& McDevitt, M. (2011). The roots of the gender gap in political knowledge in adolescence. Political Behavior, 33(3), 505-533. https://doi.org/10.1007/s11109-010-9142-9

Zamarrón, I. (2017, marzo 7). México, en busca de ingenieras. Publímetro. https://www.publimetro.com.mx/mx/noticias/2017/03/07/ mexico-busca-ingenieras.html 